\title{
The Outcome of Patients with Acute Coronary Syndrome Those Associated with Impaired Glucose Tolerance in AL-Nasiriya Governorate / Iraq
}

\author{
Basim Audib Motar \\ Department of Internal Medicine, Thi-Qar University, Thi-Qar, Iraq \\ Email address: \\ dr.basim_moter@yahoo.com

\section{To cite this article:} \\ Basim Audib Motar. The Outcome of Patients with Acute Coronary Syndrome Those Associated with Impaired Glucose Tolerance in AL- \\ Nasiriya Governorate / Iraq. American Journal of Health Research. Vol. 6, No. 5, 2018, pp. 112-118. doi: 10.11648/j.ajhr.20180605.11
}

Received: September 21, 2017; Accepted: October 28, 2017; Published: December 4, 2018

\begin{abstract}
Prediabetes in the form of impaired glucose tolerance (IGT) is common among patients with acute coronary syndrome (ACS). IGT in patients admitted for ACS is associated with increased mortality. We aimed to determine the prevalence of prediabetes in the form of IGT among ACS patients who were known to be neither diabetic nor prediabetic and referred to early coronary angiography. The study included 284 participants. We divided into 2 groups: patients group and control group. The patients group were include 138 patients admitted in AL-Hussein teaching hospital with acute coronary syndrome in the form of ST-segment elevation myocardial infarction (STEMI) and non-ST-segment elevation myocardial infarction (NSTEMI). 98 patients with NSTEMI and 40 patients with STEMI. The control group were 146 participants in the form of medical staff and patients visiting the hospital with conditions other than the ischemic coronary artery disease. All participants were neither diabetic nor prediabetic. 2 hour post prandial blood sugar, blood sugar at 3 days and blood sugar after 6 weeks were done. $22 \%$ of the patients group and $6 \%$ of the control group were found to have IGT at 2 hour postprandial with highly statistically significant value ( $\mathrm{P}$ value $<0.0001$ ). $16.6 \%$ of the patients group and $7.5 \%$ of the control group were found to have IGT at 3 days after the admission with highly statistically significant value (P value $<0.001) .11 \%$ of the patients group and $2 \%$ of the control group were found to have IGT after 6 weeks of the presentation with also significant statistical value (P value $<0.005$ ). IGT was found in $62 \%$ of the female and $38 \%$ of the male in patients group. $42 \%$ of NSTEMI and $28 \%$ of STEMI patients were ended with early percutaneous coronary angiography. There were 11 patient with IGT in the patients group died during the first week of presentation in comparison to 2 patients with normal sugar died during the same period with highly statistically significant value (P value $<0.0001$ ). IGT is common in patients admitted with ACS. IGT is associated with higher mortality among those patients with previously unreported as diabetes mellitus and should be screened for.
\end{abstract}

Keywords: Acute Coronary Syndrome, Impaired Glucose Tolerance, Mortality, Coronary Angiography

\section{Introduction}

Impaired glucose tolerance and impaired fasting glucose form an intermediate stage in the natural history of diabetes mellitus [1]. Impaired fasting glucose (IFG) is defined as fasting plasma glucose values of $6.1-6.9 \mathrm{mmol} / \mathrm{L}$ and impaired glucose tolerance (IGT) is defined as 2 hour postprandial plasma glucose of 7.8-11.0 mmol/L [2]. Type 2 diabetes mellitus is a major health problem that is associated with significant mortality and morbidity [3]. Diabetes can be prevented or delayed through lifestyle interventions $[4,5]$. Information that has been learned about the natural history and pathogenesis of diabetes indicates that this disease has a prolonged prediabetic phase [6]. The categories of impaired glucose tolerance (IGT) and impaired fasting glucose (IFG) have been officially termed prediabetes, because they are risk factors for future diabetes and for cardiovascular disease [7, 8]. Patients with impaired glucose tolerance (IGT) are at significant risk for diabetes $[9,10]$. Both prediabetes and diabetes highly predispose to cardiovascular alterations [11]. Type 2 diabetes mellitus, a presently rapidly expanding disease [12], is a major risk factor for cardiovascular morbidity and mortality [13]. Established diabetes is associated with impaired prognosis after myocardial 
infarction [11].

Newly detected abnormal glucose tolerance is one of the strongest prognostic factors following an myocardial infarction [14]. Strict insulin-based glucometabolic control improves survival in patients with diabetes and acute myocardial infarction [15]. Diabetes is also associated with worse outcomes of revascularization in the form of increased instent restenosis and increased major adverse cardiac events in percutaneous coronary intervention (PCI) and coronary artery bypass grafting (CABG) [16].

Very recently fasting blood glucose was found to be an independent risk factor for poor long term outcome and was associated with enhanced platelet reactivity and low response to clopidogrel in patients with acute coronary syndrome (ACS) and poor long term outcome of PCI [17]. Each 1\% increase in glycated haemoglobin ( $\mathrm{HbA} 1 \mathrm{c})$ level was associated with a $14 \%$ increase in the incidence of fatal and nonfatal myocardial infarction [18]. A meta-analysis of several observational studies showed that the association of cardiovascular risk with post-challenge glucose concentration is stronger than that of fasting plasma glucose [19]. These data are supported by the Diabetes epidemiology: collaborative analysis of diagnostic criteria in Europe (DECODE), which showed that 2-hour glucose, but not fasting glucose, predicts cardiovascular disease mortality in individuals with glucose levels within normal range [17]. It still unknown which underlying metabolic abnormalities that cause the increased cardiovascular disease risk in people with elevated 2-hour glucose, the 2-hour glucose is closely related to peripheral insulin resistance and lack of beta cell compensation [20].

Prediabetes ( impaired glucose tolerance) develops for the same reasons as type 2 diabetes [21]. There are various risk factors can increase the risk of developing prediabetes, these include: being overweight or obese, having a close family history of diabetes, little physical activity, previous history of gestational diabetes [22].

\section{Aim of the Study}

The aim of this study to determine the prevalence of impaired glucose tolerance in those patients with acute coronary syndrome who were known to be neither diabetic nor prediabetic and to know the outcome of those patients.

\section{Method}

\subsection{Patients}

The study was carried out on 284 participants. They were divided into 2 groups:

Group 1 (patients group): 138 patients presenting in coronary care unit in AL-Hussein teaching hospital from January 2017 - June 2017 with a new ischemic events in the form of acute coronary syndrome (ACS) which include STEMI (ST- segment elevation myocardial infarction) and NSTEMI (non-ST segment elevation myocardial infarction and unstable angina) were diagnosed according to the clinical, physical, ECG and laboratory findings.

There were 98 patients with NSTEMI and 40 patients with STEMI.

Group 2 (control group): 146 person who were medical staff or patients presenting to the outpatient clinic in ALHussein teaching hospital with condition other than the ischemic heart disease.

All the 284 participants were non diabetic (no previous diagnosis of diabetes nor prediabetics). Their ages range from 40-75 years. The participants were subjected to detailed medical history, clinical examination and ECG. Laboratory analyses including fasting blood sugar, 2 hour post prandial blood sugar, cardiac markers, renal function test and lipid profiles were withdrawn from the patients.

\subsection{Materials}

The patients with impaired glucose tolerance (IGT) at 2 hour post prandial sugar were followed by measurement of blood sugar after 3 days and then 6 weeks from presentation to document the true glucose intolerance from stress induced hyperglycemia. Glucose monitoring was stopped when the glucose level remain $<7.8 \mathrm{mmol} / \mathrm{L}$ [23].

The therapy for those patients with acute coronary syndrome after the usual treatment in coronary care unit were based on TIMI (Thrombolysis in Myocardial Infarction) score risk which is a simple prognostication scheme that categorize a patient's risk of death and ischemic events and provides a basis for therapeutic decision making [24].

TIMI Risk Score Calculation (1 point for each) include:

1. Age $\geq 65$ year.

2. Aspirin use in the last 7 days (patient experience chest pain despite aspirin use in past 7 days).

3. At least 2 anginal episodes within the last 24 hours.

4. ST changes of at least $0.5 \mathrm{~mm}$ in contiguous leads.

5. Elevated serum cardiac biomarkers.

6. Known coronary artery disease (coronary stenosis $\geq$ $50 \%)$.

7. At least 3 risk factors for coronary artery disease, such as:

a. Hypertension, blood pressure $\geq 140 / 90$ or on antihypertensive medications.

b. Current cigarette smoker.

c. Low HDL cholesterol $(<40 \mathrm{mg} / \mathrm{dL})$.

d. Family history of premature coronary artery disease, include:

A. Male first-degree relative or father younger than 55 year.

B. Female first-degree relative or mother younger than 65 year.

Score interpretation:

1. Score of $0-1=407 \%$ risk

2. Score of $2=8.3 \%$ risk

3. Score of $3=13.2 \%$ risk

4. Score of $4=19.9 \%$ risk

5. Score of $5=26.2 \%$ risk

6. Score of $6-7=$ at least 40.9 risk 
Drug therapy was appropriate for those patients with low TIMI risk score. Early coronary angiography and revascularization were considered for those patients with medium to high TIMI risk score and those with low TIMI score risk who failed to settle with drug therapy.

The coronary angiography were done using standard techniques in AL-Nasiriya cardiac center.

\section{Result}

Table 1 show the characteristics of the study.

The mean age of all patients group in this study was $58 \pm$ 11. It was noticed that $62 \%$ of the included patients were females while $38 \%$ were males. It was also noticed that the mean BMI among the patients $(28 \pm 4.2)$ was higher than the control group (23 \pm 4.6$)$. Smoker were found to be $38.4 \%$ among the patients in contrary to $31.2 \%$ in the control group. Hypertensive patients were $62.5 \%$ among the patients group while $36.2 \%$ of the control group were found to be hypertensive.

Table 1. The characteristics of the study.

\begin{tabular}{lll}
\hline characteristics & Group 1 (patients) & Group 2 (control) \\
\hline Age (years) & $58 \pm 11$ & $51 \pm 12$ \\
Gender & $62 \%$ female & $54 \%$ female \\
& $38 \%$ male & $46 \%$ male \\
BMI & $28 \pm 4.2$ & $23 \pm 4.6$ \\
Hyoking & $38.40 \%$ & $31.20 \%$ \\
\hline
\end{tabular}

Figure 1 shows the percentage of types of ACS in patients group

It was noticed that $60 \%$ of patients were admitted with non-ST-segment elevation myocardial infarction and unstable angina (NSTEMI) while $40 \%$ of the patients were found to have ST-segment elevation myocardial infarction (STEMI) as show in figure 1.

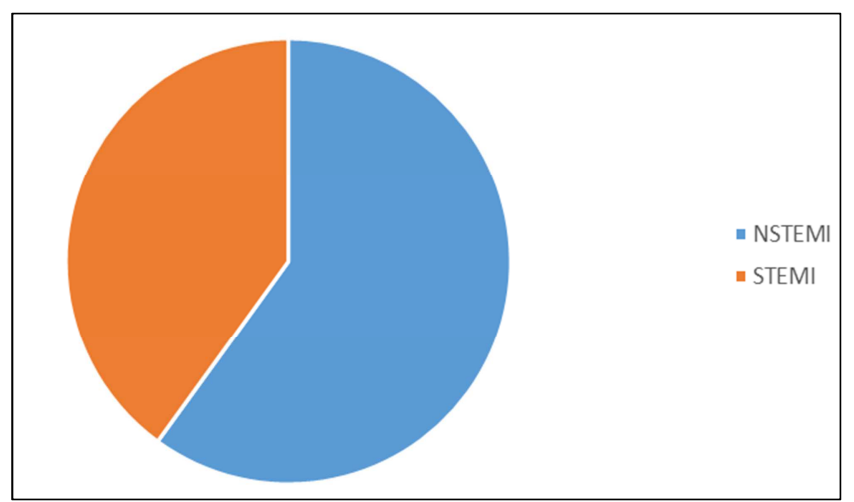

Figure 1. Percentage of the types of ACS in patients group.

$22 \%$ of the patient's group were found to be have impaired glucose tolerance (IGT) at 2 hour post prandial while only $6 \%$ of the control group found to be have IGT at the same period and that was statistically significant (P value $<0.0001$ ), as shown in figure 2 .

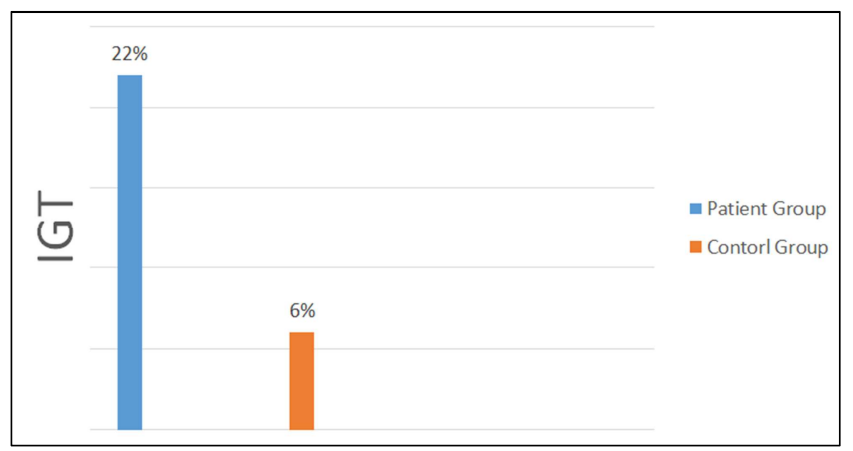

Figure 2. The percentage of participants with impaired glucose tolerance $(I G T)$ at 2 hour post prandial for patients and control group (P value < $0.0001)$.

$16.6 \%$ of the patient's group were found to be have impaired glucose tolerance (IGT) at day 3 while only $7.5 \%$ of the control group found to be have IGT at the same period and that was statistically significant ( $\mathrm{P}$ value $<0.001$ ), as shown in figure 3.

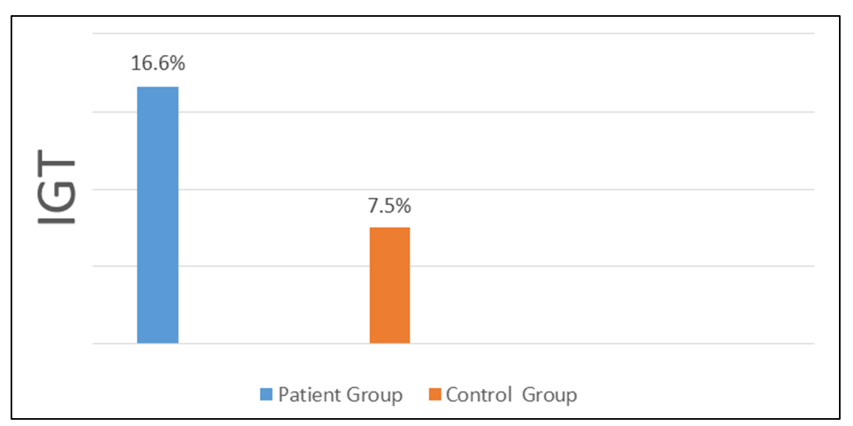

Figure 3. The percentage of participants with impaired glucose tolerance (IGT) at day 3 for patients and control group. (P value $<0.001)$.

$11 \%$ of the patients group were found to be have impaired glucose tolerance (IGT) after 6 weeks while only $2 \%$ of the control group found to be have IGT at the same period and that was statistically significant $(\mathrm{P}$ value $<0.005)$, as shown in figure 4 .

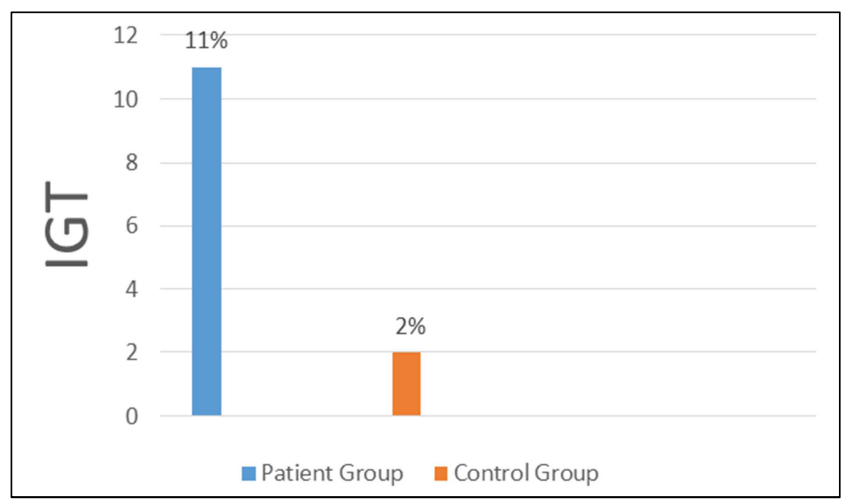

Figure 4. The percentage of IGT after 6 weeks in patients and Control group (P value $<0.005)$.

$62 \%$ of the female in the patients group and $38 \%$ of the male in the same group were found to have impaired glucose tolerance (IGT) with statistically significant (P value $<0.05$ ), 
as shown in figure 5 .

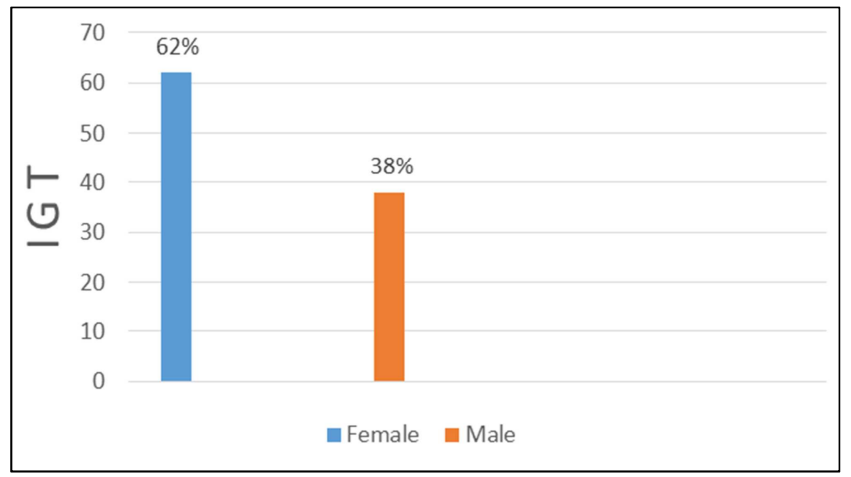

Figure 5. Percentage of IGT regarding the gender of the patients group $(P$ value $<0.05$ )

Figure 6 show $42 \%$ of patients with NSTEMI (non STSegment elevation myocardial infarction and unstable angina) and $28 \%$ of patients with STEMI were ended with early percutaneous coronary angiography (PCI) in the cardiac center, as shown in the figure below:

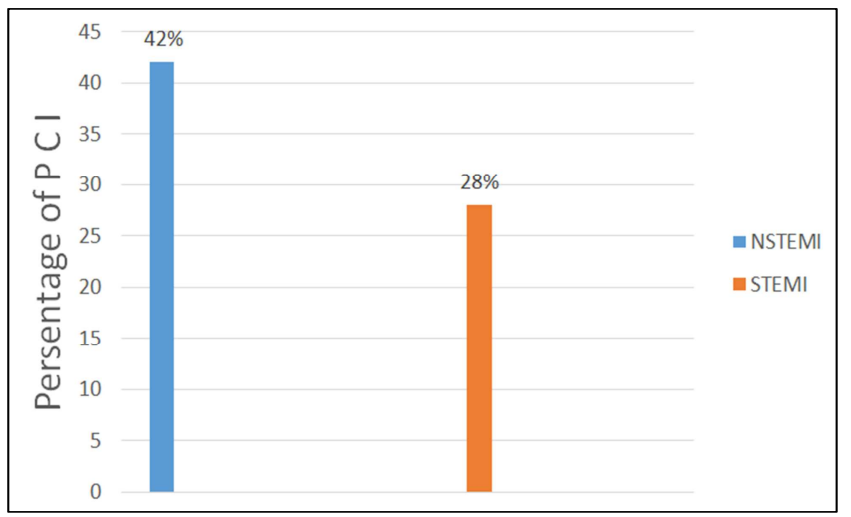

Figure 6. Percentage of patients were ended with PCI in the cardiac center.

11 patients with ACS those found to be have impaired glucose tolerance (IGT) were died during the first week of presentation in comparison with 2 patients with normal sugar state were died in that period of presentation with highly statistically significant value $(P$ value $<0.0001)$ as shown in figure 7.

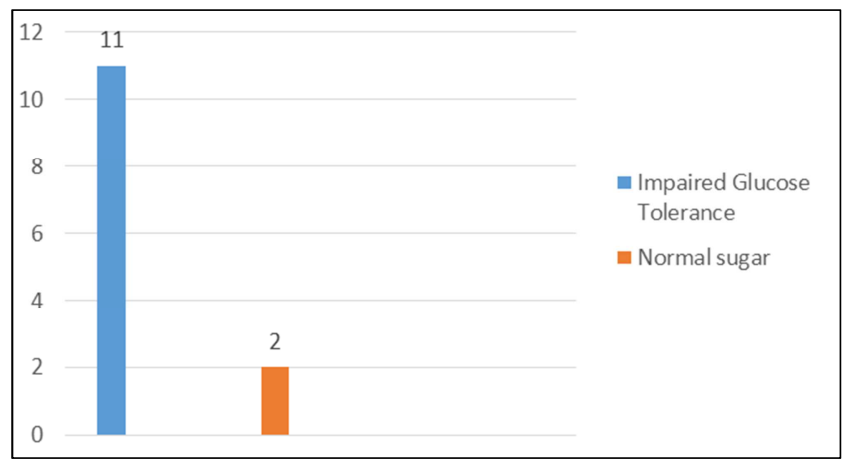

Figure 7. The number of deaths of the ACS patients with it's correlation to IGT during the first week $(P<0.0001)$.

\section{Discussion}

Impaired glucose tolerance (IGT) identify individuals at increased risk for developing diabetes [25]. It has been suggested that IGT and IFG are associated with varying rates of progression to diabetes and differences in cardiovascular disease risk [25].

In this study, we choose 284 participants who were known to be neither diabetic nor prediabetic and we divided into 2 groups: group 1 which include 138 patients were admitted in the AL-Hussein teaching hospital with ACS in the form of 98 patients with the diagnosis of NSTEMI (non-ST-segment elevation myocardial infarction and unstable angina) and 40 patients with the diagnosis of STEMI (ST-segment elevation myocardial infarction). Group 2, which was the control group and include 146 participants (medical staff and patients visiting the outpatient clinic in the AL-Hussein teaching hospital for medical advice for conditions rather than ischemic artery disease.

We found that $62 \%$ of the patients group were female while $38 \%$ of the same group were male. The burden of ACS is high in women. V. L. Roger, et al. [26] show women have higher rates of angina than do men. A female excess of angina prevalence was also demonstrated in a meta-analysis of data from 31 widely varied countries, including nonEnglish speaking countries [27].

On cardiovascular computed tomography, women have been shown to have smaller coronary artery diameters than men do [28].

Recently, disorders of the coronary microvasculature and endothelial dysfunction have been implicated in the occurrence of coronary artery disease in women. Han et al. [29] studied women and men with early coronary artery disease and found that women have more disease of the microvasculature.

This study also revealed that the mean age was $58 \pm 11$ year in the patients group while $51 \pm 12$ year in the control group, BMI was $28 \pm 4.2$ in the patients group and $23 \pm 3.6$ in the control group with no clear differences between them. Smoking was documented in $38.4 \%$ in the patients group while in the control group was found to be $31.2 \%$.

Hypertension was detected in $62.5 \%$ of the all patients group while $36.2 \%$ of the control group were found to be have hypertension. This finding correlate with other study, J. Spinar, et al. which found that the hypertension found in the majority of the patients with CAD were studied and found also that the hypertension doubles the risk of cardiovascular disease and accelerates significantly the development of atherosclerosis [30].

Figure 1 show that $60 \%$ of the patients group was NSTEMI (non-ST-segment elevation myocardial infarction and unstable angina) and $40 \%$ of the patients group was STEMI (ST-segment elevation myocardial infarction). Epidemiological data have shown that acute coronary syndrome (ACS) cases with STEMI appear to be declining and that NSTEMI occurs more frequently than STEMI [31, 32]. In the United States, it is estimated that $>780000$ people 
will experience an ACS each year, and approximately $70 \%$ of these will have NSTEMI [33].

Trends from the world's largest database of patients with ACS show that the percentage of patients with a diagnosis of NSEMI is rising dramatically [34]. This is likely to be due to the advent of more sensitive assays for myocardial injury, earlier pharmacotherapy, and reperfusion (and prevention) of STEMI [34, 35].

Figure 2 show that $22 \%$ of the patients group and $6 \%$ of the control group were found to be have impaired glucose tolerance (IGT) with statistically significant at 2 hour post prandial blood glucose and this correlate with a study, $\mathrm{M}$. Bartnik, et al. which revealed about $25 \%$ of the acute coronary artery disease had IGT [36]. In China Heart Survey aimed at characterizing the glucometabolic state of patients with CAD with no history of diabetes revealed $32.6 \%$ of those patients had isolated IGT [37]. Jitender Mokta, et al. study showed a high prevalence of IGT $(62 \%)$ in patients admitted with ACS in mountainous state on India [38].

Elevated plasma glucose levels on admission are very common in patients with acute myocardial infarction and can be the first indication of glucose intolerance [39]. It is important to mention that more than $50 \%$ of IGT and stress hyperglycemia at admission had IGT on repeat test at further time [40], this finding was consistent with our study as shown in figure 3 and figure 4 , which revealed $16.6 \%$ of the patients group and $7.5 \%$ of the control group had IGT at 3 days with statistically significant $(\mathrm{P}$ value $<0.001)$ and $11 \%$ of the patients group and $2 \%$ of the control group had IGT after 6 weeks with also statistically significant ( $\mathrm{P}$ value < $0.005)$.

The present results on the prevalence of IGT among the patients are in agreement with several recent reports. Meier et al., who found $24 \%$ of the patients had IGT after 4 weeks from the onset of ACS [41].

Figure 5 show that $62 \%$ of the female in the patients group and $38 \%$ of the male in the same group were found to have impaired glucose tolerance (IGT) with statistically significant $(\mathrm{P}$ value $<0.05)$. These results are in agreement with Annika Dotevall et al., who found that the majority of patients with ACS have abnormal glucose metabolism and that women are significantly more likely to have diabetes or IGT than men [42]. A heavier risk factors burden and a stronger effect of risk factors in diabetic and nondiabetic women than men might, at least partly, influence the effect on cardiovascular risk in women [43].

Figure 6 show $42 \%$ of patients with NSTEMI (non STSegment elevation myocardial infarction and unstable angina) and $28 \%$ of patients with STEMI were ended with early percutaneous coronary angiography (PCI) in the cardiac center. This result is differ from other study which revealed that the PCI was more common in STEMI patients compared with NSTEMI patients who had either coronary artery bypass grafting or medical management [44].

Figure 7 show that 11 patients with ACS those found to be have impaired glucose tolerance (IGT) were died during the first week of presentation in comparison with 2 patients with normal sugar state were died in that period of presentation with highly statistically significant value ( $\mathrm{P}$ value $<0.0001$ ) which similar to a study, Prashanth Panduranga. et al. which demonstrate that IGT levels are associated with increased mortality in nondiabetic ACS patients [45].

The disparity in in-hospital mortality between ACS patients with IGT and normal sugar is not clearly elucidated. Various causes have been postulated to explain this disparity. Secondary to hyperdrenergic state following ACS, stress-related relative insulin deficiency develops leading to stress hyperglycemia [46]. Cardiovascular stress induces release of catecholamines, cortisol, and glucagon, leading to increases in glucose and free fatty acids that enhances hepatic gluconeogenesis, diminish peripheral glucose uptake, and decrease myocardial glucose utilization, all of which may have adverse effects on myocardial energy metabolism and function the presence of ischemia [46].

\section{Conclusion}

Prediabetic state, defined as IGT, was associated with an increased risk of cardiovascular disease in the form of acute coronary syndrome (ACS) and also associated with higher mortality in those patients comparing with ACS patients with normal sugar state.

\section{References}

[1] Knowler WC, Barrett-Connor E, Hamman RF, Lachin JM, Walker EA, et al. Reduction in the incidence of type 2 diabetes with lifestyle intervention or metformin. $\mathrm{N}$ Engl $\mathrm{J}$ Med. 2002; 346: 393-403.

[2] S. Genuth, K. G. Alberti, P. Bennett, J. Buse, R. Defronzo, R. Kahn, et al. Follow-up report on the diagnosis of diabetes mellitus. Diabetic Care, 26 (2003), pp. 3160-3167.

[3] Shobha S. Rao, Philip D, Tamara M. Impaired glucose tolerance and impaired fasting glucose. Am Fam Physician. 2004 Apr 15; 69 (8): 1961-1968.

[4] Pan XR, Li GW, Hu YH, Wang JX, Yang WY, An ZX, et al. Effects of diet and exercise in preventing NIDDM in people with impaired glucose tolerance. The Da Qing IGT and Diabetes Study. Diabetes Care. 1997; 20: 537-44.

[5] Tuomilehto J, Eriksson JG, Valle TT, Hamalainen H, IIanneParikka P, et al. Prevention of type 2 diabetes mellitus by changes in lifestyle among subjects with impaired glucose tolerance. N Engl J Med. 2001; 344: 1343-50.

[6] Ramlo-Halsted BA, Edelman SV. The natural history of type 2 diabetes. Implications for clinical practice. Prim Care. 1999; 26: 771-89.

[7] Faerch K, Vaag A, Holst JJ, Pedersen O, Borch-Johnsen K. Impaired fasting glycemia VS impaired glucose tolerance: similar impairment of pancreatic alpha and beta cell function but differential roles of incretin hormones and insulin action. Diabetologia. 2008 May. 51 (5): 853-61. 
[8] Nathan DM, Davidson MB, Defronzo RA, Heine RJ, Henry $\mathrm{RR}$, et al. Impaired fasting glucose and impaired glucose tolerance: Implications for care. Diabetes care. 2007 Mar. 30 (3): 753-9.

[9] Capes S, Anand S. What is type 2 diabetes? In: Gerstein HC, Haynes RB, eds. Evidence-based diabetes care. Hamilton, Ont.; Decker, 2001: 151-63.

[10] Kanaya AM, Narayan KM. Prevention of type 2 diabetes: data from recent trials. Prim Care. 2003; 30: 511-26.

[11] Dotevall Annika, Rosengren Annika, Bartnik Malgorzata, Malmberg Klas, Ohrvik John, Simoons Maarten, et al. On behalf of the Euro Heart Survey Investigations. Sex-related aspects on abnormal glucose regulation in patients with coronary artery disease. Eur Heart J (2007), pp. 310-315.

[12] King H, Aubert RE, Herman WH. Global burden of diabetes 1995-2025. Prevalence, numerical estimates and projections. Diabetes Care. 1998: 21: 1414-1431.

[13] Haffner SM, Lento S, Ronnemaa T, et al. Mortality from coronary heart disease in subjects with type 2 diabetes and in non diabetic subjects with and without prior myocardial infarction. N Engl J Med. 1999; 339: 229-234.

[14] Bartnik M, Malmberg K, A Norhammar, et al. Newly detected abnormal glucose tolerance important predictor of long term outcome after an acute myocardial infarction. Eur Heart J. 2004; 25 Abstract Supplement, Abstract no.81979.

[15] Malmberg K, Ryden L, Efendic S, et al. Randomized trial of insulin-glucose infusion followed by subcutaneous insulin treatment in diabetic patients with acute myocardial infarction (DIGAMI study): effects on mortality at 1 year. J Am Coll Cardiol. 1995; 26: 57-65.

[16] Carlos Cuellas, Antonio De Miguel, Alejandro Diego, Felipe Fernadez-Vazquez, et al. Fasting glucose is an independent risk factor for long-term outcome and is associated with enhanced platelet reactivity and low response to clopidogrel in patients with acute coronary syndrome. Euro PCR, Abstract, 2009, E 120.

[17] Stratton IM, Adler AI, Neil HA, Matthews DR, Manley SE, Hadden D, Turner RC, Holman RR. Association of glycemia with macrovascular and microvascular complications of type 2 diabetes (UKPDS 35): prospective observational study. British Medical Journal. 2000; 321: 405-412.

[18] Kodamas S, Saito K, Tanaka S, Horikawa C, Fujiwara K, et al. Fasting and Post-Challenge Glucose as Quantitative Cardiovascular Risk Factors: A Meta-analysis. J Atheroscler Thromb. 2012; 42: 926-931.

[19] Ning F, Tuomilehto J, Pyorala K, Onat A, et al. Cardiovascular Disease Mortality in Europeans in Relation to Fasting and 2-h Plasma Glucose Levels Within a Normoglycemic Range. Diabetes Care. 2010; 33: 2211-2216.

[20] Faerch K, Borch-Johnsen K, Holst JJ, Vega A. Pathophysiology and aetiology of impaired fasting glycemia and impaired glucose tolerance: does it matter for prevention and treatment of type 2 diabetes. Diabetologia. 2009; 52: 1714-1723.

[21] Bar EL, Zimmet PZ, Welborn TA, et al. Risk of cardiovascular and all-cause mortality in individuals with diabetes mellitus, impaired fasting glucose, and impaired glucose tolerance. Circulation. 2007; 116 (2): 151-7.
[22] Raina Elley C, Kenealy T. Lifestyle interventions reduced the long-term risk of diabetesin adults with impaired glucose tolerance. Evid Based Med. 2008 (December); 13 (6): 173.

[23] Umpierrez GE, Hellman R, Korytkowski MT, Kosiborod M, Maynard GA, Montori VM, et al. Management of Hyperglycemia in Hospitalized Patients in Non-critical Care Setting: An Endocrine Society Clinical Practice Guideline. Journal of Clinical Endocrinology and Metabolism.2012; 97: 16-38.

[24] Elliot M, Antman, Marc Cohen, Peter J. L. M. Bernink, Carolyn H. The TIMI Risk Score for Unstable Angina/NonST Elevation MI. Jama. 2000; 284 (7): 835-42.

[25] Report of the Expert Committee on the Diagnosis and Clsssification of Diabetes Mellitus. American Diabetes Association; the expert committee on the diagnosis of classification of Diabetes Mellitus. Diabetes Care.1997; 20: 1183-97.

[26] V. L. Roger, A. S. Go, D. M. LIoyd-Jones, et al. American Heart Association Statistics Committee and Stroke Statistics Subcomittee. Heart disease and stroke statistics - 2012 update: a report from the American Heart association. Circulation. 2012; 125: pp. e2-220.

[27] H. Hemingway, C. Lanfenberg, J. Damant, C. Frost, K. Pyorala, E. Barrett-Conor. Prevalence of angina in women versus men: a systematic review and meta-analysis of international variations across 31 countries. Circulation. 2008; 117: $1526-1536$.

[28] J. A. Dickerson, H. N. Nagaraja, S. V. Raman. Gender-related differences in coronary artery dimensions: a volumetric analysis. Clin Cardiol. 2010; 33: 44-49.

[29] S. H. Han, J. H. Bae, D. R. Holmes Jr., et al. Sex differences in atheroma burden and endothelial function in patients with early coronary atherosclerosis. Eur Heart J. 2008; 29: 13591369 .

[30] J. Spiner, J. Vitovec, et al. Ischemic heart disease. Grada Publishing, Prasha (2003).

[31] J. P. Bassand, N. Danchin, G. Filippatos, et al. Implementation of reperfusion therapy in acute myocardial infarction. A policy statement from the Eur Heart society. Eur Heart J. 2005; 26: 2733-2741.

[32] G. Montalescot, J. Dallongeville, E. V. Belle, et al. STEMI and NSTEMI: are they so different? 1 year outcomes in the acute myocardial infarction as defined by ESC/ACC definition. Eur Heart J. 2007; 28: 1409-1417.

[33] J. G. Canto, M. G. Shlipak, W. J. Rogers, et al. Prevalence, clinical characteristics, and mortality among patients with myocardial infarction presenting without chest pain. J Am Med Assoc. 2000; 283: 3223-3229.

[34] W. J. Rogers, P. D. Frederick, E. Stoehr, et al. Trends in presenting characteristics and hospital mortality among patients with ST elevation and non-ST elevation myocardial infarction in the National registry of myocardial infarction from 1990 to 2000. Am Heart J. 2008; 156: 1026-1034.

[35] I. Eitel, A. Franke, G. Schuler, H. Thiele. ST-segment resolution and prognosis after facilitated versus primary percutaneous coronary intervention in acute myocardial infarction. Clin Res Cardiol. 2010; 99: 1-11. 
[36] M. Bartnik, L. Ryden, R. Ferrari, K. Malmberg, Pyorala, M. Simoons, et al. On behalf of the Euro Heart Survey Investigators. The prevalence of abnormal glucose regulation in patients with coronary artery disease across Europe. Eur Heart J. 2004; 25: 1880-1890.

[37] The China Heart Survey. Eur Heart J. 2006; 27 (21): 2573-79.

[38] Jitender Mokta, Subash Kumar, Neeraj Ganju, et al. High incidence of abnormal glucose metabolism in acute coronary syndrome patients at a moderate altitude: A sub-Himalayan study. Indian J Endocrinol Metabo. 2017; 21 (1): 142-147.

[39] De Mulder M, Oemrawsingh RM, Stam F, et al. Comparison of diagnostic criteria to detect undiagnosed diabetes in hyperglycemia patients with acute coronary syndrome. Heart. 2012; 98: 37-41.

[40] Tuomilehto J, Lindstrom J, Eriksson LG, et al. Prevention of type 2 diabetes mellitus by changes in lifestyle among subjects with impaired glucose tolerance. N Engl J Med. 2001; 344: 1343-50.

[41] Meier JJ, Deifluss S, Gallwitz B, KlamannA, et al. Influence of impaired glucose tolerance on long term survival after acute myocardial infarction. The LAngendreer Myocardial infarction and blood glucose in diabetic patients Assessment (LAMBDA). Dtsch Med Wochenschr. 2002; 127: 1123-9.
[42] Annika Dotevall, Annika Rosengren, Malgorzata Bartnik, et al. Sex-related aspects on abnormal glucose regulation in patients with coronary artery disease. European Heart Journal. 2007; 28: 310-315.

[43] Juutilainen A, Kortelainen S, Lehto S, Ronnemaa T, et al. Gender difference in the impact of type 2 diabetes on coronary heart disease risk. Diabetes Care. 2004; 27: 2898-2904.

[44] Vora AN, Wang TY, Hellkamp AS, et al. Older patients with ST-elevation vs non-ST-elevation myocardial infarction with angiographically proven coronary artery disease. Circ Cardiovasc Qual Outcomes. 2016; 9.

[45] Prashanth Panduranga, Kadhim Sulaiman, Jawad Al-Lawati, et al. Relationship Between Admitting Nonfasting Blood Glucose and In-Hospital Mortality Stratified by Diabetes Mellitus Among Acute Coronary Syndrome Patients in Oman. Heart Views. 2011; 12 (1): 12-17.

[46] Stranders I, Diamant M, van Gelder RE, Spruijt HJ, et al. Admission blood glucose level as risk indicator of death after myocardial infarction in patients with and without diabetes mellitus. Arch Intern Med. 2004; 164: 982-98. 